\title{
Deletion of Hyaluronan-Binding Protein Involved in Hyaluronan Depolymerization (HYBID) Results in Attenuation of Osteoarthritis in Mice
}

\author{
Masahiro Momoeda, ${ }^{* \dagger}$ Susana de Vega, ${ }^{*}$ Haruka Kaneko, ${ }^{\dagger}$ Chiho Yoshinaga, ${ }^{*}$ Masayuki Shimoda, ${ }^{\ddagger}$ Tomomi Nakamura, \\ Yoko Endo, ${ }^{\S}$ Hiroyuki Yoshida, ${ }^{\S}$ Kazuo Kaneko, ${ }^{* \dagger}$ Muneaki Ishijima, ${ }^{* \dagger}$ and Yasunori Okada*
}

From the Departments of Pathophysiology for Locomotive and Neoplastic Diseases* and Medicine for Orthopaedics and Motor Organ, ${ }^{\dagger}$ Juntendo University Graduate School of Medicine, Tokyo; the Department of Pathology, ${ }^{\ddagger}$ Keio University School of Medicine, Tokyo; and the Biological Science Research, ${ }^{\S}$ Kao Corporation, Kanagawa, Japan

Accepted for publication

July 28, 2021.

Address correspondence to Yasunori Okada, M.D., Ph.D., Department of Pathophysiology for Locomotive and Neoplastic Diseases, Juntendo University Graduate School of Medicine, 2-1-1 Hongo, Bunkyo-ku, Tokyo 113-8241, Japan. Email: ya-okada@juntendo.ac.jp.

\begin{abstract}
Hyaluronan (HA)-binding protein involved in HA depolymerization (HYBID) is involved in cartilage destruction via HA depolymerization in human knee osteoarthritis. However, the role of HYBID in the progression of osteoarthritis remain elusive. This study sought to examine whether genetic depletion of Hybid could suppress surgically induced osteoarthritis of mouse knee joints. In osteoarthritis induced by medial collateral ligament transection with meniscus removal, articular cartilage destruction and osteophyte formation at the medial femoral-tibial joint were significantly inhibited in Hybid-deficient $\left(\mathrm{Hybid}^{-1-}\right)$ mice compared with wild-type mice. Hybid was highly produced by synovial cells and articular chondrocytes in the osteoarthritis joints of wild-type mice. IL-1 $\beta$, IL-6, and tumor necrosis factor- $\alpha$ were up-regulated in the osteoarthritis joint tissues of both wild-type and $\mathrm{Hybid}^{-1-}$ mice. Vascular density at the synovial and periosteal junction was significantly reduced in $\mathrm{Hybid}^{-1-}$ mice compared with wild-type mice. High-molecular-weight HA accumulated in osteoarthritis joint tissues of $\mathrm{Hybid}^{-1-}$ mice. Injections of high-molecular-weight HA to knee joints attenuated the cartilage destruction and osteophyte formation in wild-type mouse osteoarthritis group. Inhibition of cartilage destruction and osteophyte formation in $\mathrm{Hybid}^{-{ }^{-}}$mice was also observed in destabilization of the medial meniscus model. These data are the first to demonstrate that cartilage destruction and osteophyte formation are suppressed in $\mathrm{Hybid}^{-/-}$mice and suggest that Hybid-mediated HA depolymerization is implicated for the progression of mechanically-induced knee osteoarthritis. (Am J Pathol 2021, 191: 1986-1998; https://doi.org/10.1016/j.ajpath.2021.07.008)
\end{abstract}

Osteoarthritis (OA) is the most common joint disease characterized by the destruction of articular cartilage, osteophyte formation, synovitis, and subchondral bone changes. Although OA affects all joints, knee joint is the most common site of OA. ${ }^{1}$ Though several risk factors for knee joint OA include age, obesity, genetic predisposition, joint malalignment, trauma, sex, and occupational overuse, ${ }^{1-3}$ he abnormal mechanical loading to the knee joint may be a major inducer for knee OA. ${ }^{2}$ Continuous mechanical stress in subjects with meniscal tears, joint malalignment, and/or obesity commonly leads to OA of the knee joint, showing destruction of articular cartilage and osteophyte formation. ${ }^{2}$ On the basis of these clinical findings, knee OA models have been developed by inducing instability of knee joints through surgical intervention in both large animals, such as the dog, goat/sheep, and horse, and smaller animals, such as the mouse, rat, rabbit, and guinea pig. ${ }^{4}$ Among these, mouse models are commonly used

Supported by Grant-in-Aid for Scientific Research (JSPS KAKENHI) grants 18K09082 (M.I.), 16H05454 (Y.O.), and 19H03788 (Y.O.) from Japan Society for the Promotion of Science.

Disclosures: T.N., Y.E., and H.Y. are employees of Kao Corp.; H.Y. is a holder of the HYBID (hyaluronan-binding protein involved in hyaluronan depolymerization) patent. 
to study the pathogenesis for the OA process, because genetically modified mice, including knockout mice lacking a certain target gene, can be used to understand the specific genetic contributions to the OA pathogenesis. ${ }^{4-6}$

Pathologic studies indicate that in the affected human cartilage, the hyaluronan (HA)-proteoglycan network is initially degraded followed by the digestion of collagen fibrils of type II and XI collagens. ${ }^{3,7}$ Fibrillar collagens are degraded by the proteolytic actions of the matrix metalloproteinase (MMP) family members with collagenolytic activity, which include MMP-1, MMP-13, and MMP-14, and aggrecan seems to be digested mainly by a disintegrin and metalloproteinases with thrombospondin motifs (ADAMTSs) 4 and 5 (alias aggrecanases 1 and 2, respectively). ${ }^{3,7}$ Two hyaluronidases - 1 and 2 , and a cell surface HA receptor CD44 were thought to be responsible for HA degradation. ${ }^{8,9}$ However, because knockdown of these molecules had no effect on HA degradation in human skin fibroblasts, new molecules involved in HA degradation were studied by microarray analysis of the genes in fibroblasts treated with histamine or transforming growth factor- $\beta$ (TGF- $\beta$ ), which promoted or suppressed HA degradation and identified hyaluronan-binding protein involved in hyaluronan depolymerization (HYBID) as a key enzyme for HA degradation. ${ }^{10,11}$ HYBID was originally described as a deafness gene of unknown function (KIAA1199) ${ }^{12}$ and named as CEMIP (cell migration-inducing protein). ${ }^{13}$ HYBID is a secreted molecule consisting of one G8, two GG, and four PbH1 domains, and lacks homology to hyaluronidases, HAbinding proteins, and HA-link modules. ${ }^{10}$ Murine homologue of HYBID (ie, Hybid) has been cloned. ${ }^{12}$ It has 91\% amino acid sequence homology to human HYBID and exhibits the HA binding and depolymerization activity. ${ }^{14}$ Hybid-deficient $\left(\right.$ Hybid $\left.^{-/-}\right)$mice show transiently shorter long bones secondary to delayed endochondral ossification at the growth plates, in which angiogenesis is inhibited by accumulated high-molecular-weight HA (HMW-HA). ${ }^{15}$ Transmembrane protein 2 (Tmem2), a type II transmembrane protein with a sequence homology to Hybid, is reported as a cell-surface hyaluronidase in mice. ${ }^{16} \mathrm{How}-$ ever, no information is available for changes in the expression levels of Tmem 2 and spontaneous OA formation in $\mathrm{Hybid}^{-/-}$mice or the involvement of Tmem2 in mouse OA.

HYBID is highly produced by chondrocytes located in the HA-depleted zone of the articular cartilage in human OA knee joints, ${ }^{17}$ and preliminary studies show that the expression of TMEM2 is not up-regulated in these OA cartilages (Jun Shiozawa et al, unpublished data). In addition, although TMEM2 expression is constitutive, HYBID is overexpressed by OA synovial fibroblasts compared with control normal synovial fibroblasts and related to depolymerization of HMW-HA to lower-molecular-weight HA in synovial fluid. ${ }^{18}$ These studies provide data suggesting that HYBID plays an important role in destruction of the articular cartilage by HA depolymerization in the articular cartilage and synovial fluid. However, because these were correlation studies based on the data obtained from patients with advanced knee OA, the role of HYBID in the progression of OA remains unclear.

The present study, therefore, examined the effects of Hybid-mediated HA depolymerization on the progression of OA in mechanical stress-induced mouse models. In the OA model developed by medial collateral ligament transection with meniscus removal (MCLT/M OA model), this study demonstrated that compared with wild-type mice, destruction of articular cartilage and osteophyte formation are attenuated in $\mathrm{Hybid}^{-/-}$mice, in which HMW-HA was accumulated in the joint tissues. These OA changes observed in wild-type mice are inhibited by injections of HMW-HA into knee joints. In addition, this study demonstrated similar suppressive effects on OA changes in Hybid $^{-1-}$ mice in another mild OA model.

\section{Materials and Methods}

\section{Generation and Characterization of $\mathrm{Hybid}^{-/-}$Mice}

Hybid $^{-/-}$mice were generated by deleting the exon 1 of the Hybid gene by Cre/loxP system, and the detailed information was described in our previous publication. ${ }^{15}$ Hybid-null homozygous $\mathrm{Hybid}^{-/-}$mice were generated by mating heterozygous $\mathrm{Hybid}^{+/-}$mice, and age- and sex-matched wild-type littermate mice were used as controls. The mRNA expression levels of Hybid and Tmem2 were detected with the knee joint tissues, including synovium, articular cartilage, and growth plate of femur and tibia from 1-week-old mice by quantitative real-time PCR. To study whether age-related spontaneous OA changes occur, knee joints were obtained from wild-type and Hybid ${ }^{-1-}$ mice at the age of 60 weeks and subjected to histologic examination.

\section{Knee OA Models in $\mathrm{Hybid}^{-/-}$and Wild-Type Mice}

$\mathrm{Hybid}^{-/-}$mice and wild-type littermates, at the age of 10 weeks, were anesthetized by isoflurane inhalation (Pfizer Japan Inc., Tokyo, Japan), and their left knee joints were subjected to surgery for the MCLT/M OA model by transection of the medial collateral ligament and removal of the medial meniscus, according to the methods described by Kamekura et al. ${ }^{5}$ The control right knee joints were subjected to a sham operation by the same approach, but without ligament transection and meniscectomy. Knee joints were obtained from $\mathrm{Hybid}^{-/-}$and wild-type mice at 2 and 4 weeks after the surgery, and knee joints were histologically and immunohistochemically analyzed for OA changes and Hybid protein expression. To test the effects of HMW-HA on knee OA changes, 10-week-old wild-type mice underwent MCLT/M OA surgery at both knee joints, and received intra-articular injections of $10 \mu \mathrm{L}$ of $2700-\mathrm{kDa} \mathrm{HA}$ 
(Suvenyl; Chugai Pharmaceutical Co, LTD, Tokyo, Japan) as per the clnical instructions and $10 \mu \mathrm{L}$ of saline to the left and right knee joints, respectively, at 1, 2, and 3 weeks after the surgery. They were then sacrificed at 4 weeks, and the knee joints were histologically examined.

OA changes in the medial femoral condyle and the medial tibial plateau were quantified by the Osteoarthritic Research Society International histopathologic scoring system, which consisted of structure change score with 8 degrees $(0,0.5$, and 1 to 6 ) and proteoglycan depletion score with 6 degrees (0 to 5). ${ }^{19}$ Synovitis was scored according to the method described by Lewis et al, ${ }^{20}$ which was composed of synovial lining cell layer with 4 degrees ( 0 to 3 ) and cell density with 4 degrees (0 to 3 ). Osteophyte was evaluated using osteophyte-forming scores consisting of size with 4 degrees ( 0 to 3 ) and maturity with 5 degrees ( 0 to 4 ), according to our previous study. ${ }^{21}$

The difference in OA changes in Hybid ${ }^{-1-}$ mice and wild-type littermates were examined in the destabilization of the medial meniscus (DMM) OA model, which was prepared by sectioning the medial meniscotibial ligament of the 10 -week-old mice, according to the methods by Glasson et al. ${ }^{6}$ The knee joints were histologically examined at 8 and 12 weeks after the surgery, as described above. All the experiments were performed under the NIH's Guide for the Care and Use of Laboratory Animals, ${ }^{22}$ and ethical approval was obtained from the animal ethics committee in Juntendo University (number 2020035).

\section{Histology, Immunohistochemistry, and HA Staining}

Knee joints were fixed at $4{ }^{\circ} \mathrm{C}$ in $4 \%$ buffered formaldehyde, and then decalcified with $10 \%$ EDTA in $0.1 \mathrm{~mol} / \mathrm{L}$ Tris- $\mathrm{HCl}$ buffer, $\mathrm{pH}$ 7.4. The tissues were embedded in paraffin, and then coronal sections (4 $\mu \mathrm{m}$ thick) were stained with hematoxylin and eosin and Safranin O/Fast green. Immunostaining for Hybid was performed with paraffin sections, which were subjected to antigen retrieval by autoclaving at $95^{\circ} \mathrm{C}$ for 10 minutes in Target Retrieval Solution, pH 6.0 (Dako Cytomation, Tokyo, Japan). After incubation with $3 \% \mathrm{H}_{2} \mathrm{O}_{2}$ to block peroxidase reactions and BLOCK ACE (DS Pharma Biomedical, Osaka, Japan) and goat normal serum (Vector Laboratories, Burlingame, CA) to block nonspecific reactions, they were treated with rabbit anti-KIAA1199 (HYBID/CEMIP) antibody (21129-1-AP; Proteintech, Rosemont, IL) or nonimmune control IgG (X0936; Dako Cytomation), and then incubated with biotinylated antibody against rabbit $\operatorname{IgG}$ according to the ABC method (Vector Laboratories). Color was developed using 3-amino-9-ethylcarbazole chromogen (Vector Laboratories). HA localization was determined using biotinylated HA-binding protein (Hokudo, Sapporo, Japan), according to our methods. ${ }^{15}$ Paraffin sections were treated with $3 \% \mathrm{H}_{2} \mathrm{O}_{2}$ to block endogenous peroxidase, and then incubated with $2 \mu \mathrm{g} / \mathrm{mL}$ biotinylated HA-binding protein in phosphate-buffered saline containing $10 \%$ fetal bovine serum, followed by reaction with streptavidin horseradish peroxidase (BD Biosciences, San Jose, CA). Color was developed by incubation with a solution of $3,3^{\prime}$-diaminobenzidine tetrahydrochloride (Sigma-Aldrich, St. Louis, MO), and the sections were counterstained with hematoxylin. Degree of the staining in the articular cartilage was evaluated by staining score with 4 degrees ( 0 to 3 ), in which $0,1,2$, and 3 indicated no staining, focal staining (positive in $<30 \%$ area), moderate staining (positive in $31 \%$ to $49 \%$ area), and marked staining (positive in $>50 \%$ area), respectively.

Immunostaining for CD31 was performed with paraffin sections of the knee joint tissues prepared by the AMeX $\operatorname{method}^{23}$ : The tissues were fixed in acetone at $-20^{\circ} \mathrm{C}$ overnight, cleared in methyl benzoate and xylene, and embedded in paraffin at approximately $60^{\circ} \mathrm{C}$. The sections were reacted with rat anti-CD31 monoclonal antibody (MEC13.3; BD Biosciences), and then incubated with anti-rat Fab's fragments conjugated to horseradish peroxidase-labeled amino acid polymer (Nichirei Biosciences, Tokyo, Japan). Color was developed with a solution of 3,3'-diaminobenzidine tetrahydrochloride. All the sections were counterstained with hematoxylin.

\section{Measurement of mRNA Expression}

Joint tissues, which included synovium, meniscus, and ligaments, were obtained from the knee joints of $\mathrm{Hybid}^{-/-}$and wild-type mice at 2 weeks after the surgery for MCLT/M OA model or sham operation, and total RNA was extracted from the tissues by using RNeasy Microkit (Qiagen, Alameda, CA). cDNA was synthesized with ReverTra Ace reverse transcriptase (Toyobo Life Science, Tokyo, Japan). The expression levels of Hybid, Tmem2, HA synthase 1 (Has1), Has2, Has3, Ill $\beta$, Il6, tumor necrosis factor- $\alpha$ (Tnf), Tgf $\beta$, and $C d 31$ were normalized to glyceraldehyde3-phosphate dehydrogenase (Gapdh) and determined by an SYBR Green real-time PCR assay (InvitroGen, Carlsbad, CA), according to the $\Delta \Delta \mathrm{C}_{\mathrm{T}}$ method. ${ }^{24}$ The primers' nucleotide sequences were as follows: Hybid, $5^{\prime}$-CTCCTGGCCAACTTCTCAGG-3' (forward) and $5^{\prime}$-TGCCATGGCCAATGTGTACT-3' (reverse); Tmem $2,5^{\prime}$-AGGCTGATGCCTGTATGTTGTGTC-3' (forward) and 5'-ACACGTGCCCATCCTGTGAG-3' (reverse); Has 1, 5'-GTGTCCTGCATCAGTGGTCCTC-3' (forward) and $5^{\prime}$-TGGTTGTACCAGGCCTCCAAG-3' (reverse); Has 2, 5' ${ }^{\prime}$-TGTGGCCTAAATGTGGCAGA-3' (forward) and 5'-CGCCCATGTAAACATCACTAACAG-3' (reverse); Has3, 5'-CCATGTTCTCACTCCAGCATCAA-3' (forward) and 5'-GGGCAAAGCTCAGCACCACTA-3' (reverse); Ill $\beta, 5^{\prime}$-TCCAGGATGAGGACATGAGCAC-3' (forward) and $5^{\prime}$-GAACGTCACACACCAGCAGGTTA-3' (reverse); Il6, 5' CCACTTCACAAGTCGGAGGCTTA-3' (forward) and 5'TGCAAGTGCATCATCGTTGTTC-3' (reverse); Tnf, $5^{\prime}$ ACTCCAGGCGGTGCCTATGT-3' (forward) and $5^{\prime}$-GT GAGGGTCTGGGCCATAGAA-3' (reverse); Tgf $\beta$, 5'-GT 
GTGGAGCAACATGTGGAACTCTA- $3^{\prime}$ (forward) and $5^{\prime}$ CGCTGAATCGAAAGCCCTGTA-3' (reverse); Cd31, $5^{\prime}$ CAACAGAGCCAGCAGTATGAGGAC- $3^{\prime}$ (forward) and 5'-CTGCAACTATTAAGGTGGCGATGA-3' (reverse); and Gapdh, 5'-TGTGTCCGTCGTGGATCTG-3' (forward) and $5^{\prime}$-TTGCTGTTGAAGTCGCAGG-3' (reverse).

\section{Examination of HA Content and Molecular Weight Distribution of $\mathrm{HA}$}

According to the previous methods, ${ }^{25}$ HA was extracted from the knee joint tissues, which included synovium, meniscus, and ligaments. The freeze-dried tissues were incubated at $55^{\circ} \mathrm{C}$ for 5 hours in $50 \mathrm{mmol} / \mathrm{L}$ Tris- $\mathrm{HCl}$ buffer, $\mathrm{pH} 7.4,0.5 \%$ SDS, and $100 \mathrm{mmol} / \mathrm{L}$ EDTA containing $0.2 \mathrm{mg} / \mathrm{mL}$ of proteinase K (Sigma-Aldrich). HA was isolated with phenol/chloroform, and the content was determined using a QnE Hyaluronic Acid ELISA Assay (Biotech Trading Partners, LLC, Encinitas, CA), which detected HA molecular weight forms as small as 20 to 30 $\mathrm{kDa} .{ }^{18}$ Molecular weight of purified HA samples of $0.3 \mu \mathrm{g}$ was determined by high-performance liquid chromatography using Shodex OHpak SB-807 HQ column $(0.8 \times 30$ $\mathrm{cm}$; Showa Denko, Tokyo, Japan) in $50 \mathrm{mmol} / \mathrm{L}$ phosphate buffer, $\mathrm{pH}$ 7.0. ${ }^{10}$ HA content in each fraction of $0.5 \mathrm{~mL}$ was measured by a QnE Hyaluronic Acid ELISA Assay. Calibration of the column was performed by running the following HA species: NaHA-G2 (2012 kDa; average molecular size), M2 (887 kDa), L2 (222 kDa), and S2 $(55 \mathrm{kDa})$, which were purchased from $\mathrm{PG}$ Research (Tokyo, Japan).

\section{Statistical Analysis}

Data were reported as means $\pm \mathrm{SD}$. Results between the two independent groups were determined by the two-sided $t$-test. Comparisons among more than three groups were determined by one-way analysis of variance, followed by Bonferroni post-hoc testing. $P<0.05$ was considered significant.

\section{Results}

Hybid $^{-/}$Mice Lack Tmem2 Overexpression and Development of Spontaneous Knee OA

Expression of Hybid and Tmem 2 in the knee joint tissues from 1-week-old $\mathrm{Hybid}^{-/-}$and wild-type mice showed neither expression of Hybid nor overexpression of Tmem 2 in Hybid $^{--}$compared with that in the wild-type mouse tissue (Supplemental Figure S1). Histologically, the articular cartilage of knee joints from Hybid ${ }^{-1-}$ and wild-type mice at the age of 60 weeks exhibited only minimal structural change and proteoglycan depletion, the latter of which was evaluated by Safranin O/Fast green staining (Supplemental Figure S2). The meniscus and synovium showed no definite pathologic changes, and no osteophyte formation was observed in either Hybid ${ }^{-1-}$ or wild-type mouse knee joints (Supplemental Figure S2). Histochemistry using HAbinding protein demonstrated the presence of $\mathrm{HA}$ in joint tissues, including the articular cartilage, and semiquantitative analysis of the staining showed similar scores in the articular cartilage from wild-type and $\mathrm{Hybid}^{-1-}$ mice (Supplemental Figure S3). These data indicate that the deletion of Hybid does not cause any spontaneous agerelated OA changes or excessive accumulation of HA in the mouse knee joint.

\section{Reduced Cartilage Destruction and Osteophyte Formation in Hybid ${ }^{-/}$Mouse Knee Joints in MCLT/M OA Model}

At 2 weeks after surgery for MCLT/M OA model (ie, medial collateral ligament transection with medial meniscus removal), both $\mathrm{Hybid}^{-1-}$ and wild-type mice showed definite OA changes including articular cartilage destruction and osteophyte formation at the medial femoraltibial joint (Figure 1A). These changes progressed in both groups at 4 weeks after the surgery, but they were more severe in wild-type mouse group compared with $\mathrm{Hybid}^{-/-}$ mouse group (Figure 1B). The severity of articular cartilage destruction and osteophyte formation of the tibia and synovitis was evaluated by the scoring system. Scores of structural change and proteoglycan depletion of the tibial articular cartilage and synovitis score at 2 and 4 weeks after the surgery were significantly increased in the knee joints of both wild-type and $\mathrm{Hybid}^{-/-}$mice compared with the joints that received the sham operation (Figure 1C). More important, the structural change and proteoglycan depletion scores at 2 and 4 weeks were significantly reduced in Hybid $^{-1-}$ mice compared with wild-type mice, although there were no significant changes in synovitis score (Figure 1C). Scores of the osteophyte size and maturity at 4 weeks were also significantly decreased in $\mathrm{Hybid}^{-{ }_{-}}$mice compared with wild-type mice (Figure 1C).

Expression of Hybid, Tmem2, Has1, Has2, Has3, Inflammatory Cytokines, Tgf $\beta$, and $C d 31$ in $0 \mathrm{~A}$ Joint Tissues

mRNA expression levels of Hybid, Tmem2, Has1, Has2, Has3, Ill $\beta, I l 6, T n f, T g f \beta$, and $C d 31$ were measured in the joint tissues obtained at 2 weeks after the surgery. The Hybid expression was up-regulated in wild-type mouse OA joint by 44.7-fold as compared with wild-type mouse shamoperated joint, but as expected, no expression was observed in the Hybid ${ }^{-1-}$ mouse tissues (Figure 2). The Tmem2 expression was enhanced in OA joint by 5 -fold as compared with sham-operated joint in both wild-type and Hybid ${ }^{-/}$ mouse groups, but the expression levels in OA joint were similar between the two groups (Figure 2). Has 1, Has2, and Has 3 were all significantly up-regulated in OA joint tissues 


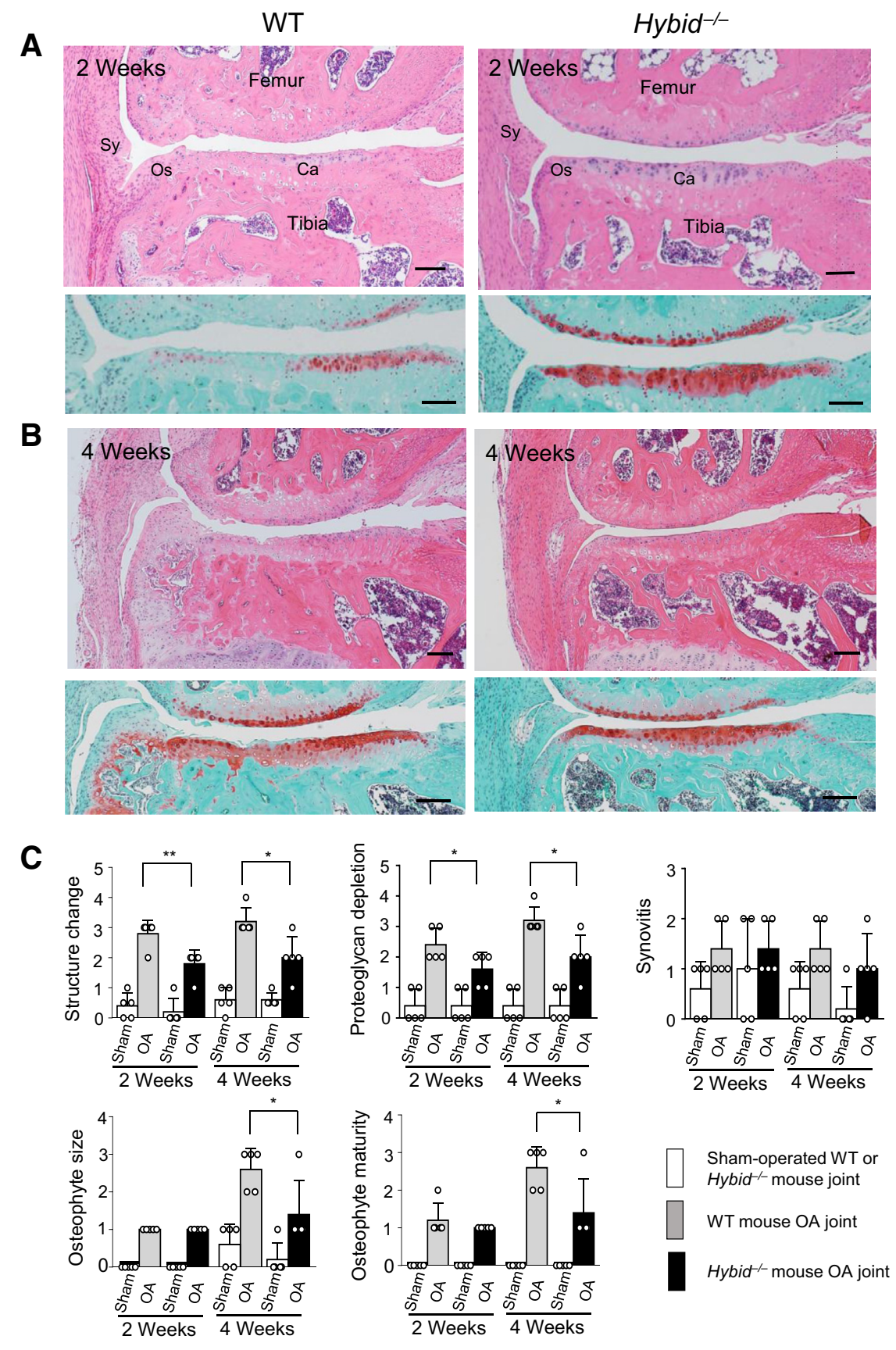

Figure 1 Histologic evaluation of the knee joints in medial collateral ligament transection with meniscus removal osteoarthritis $(0 \mathrm{~A})$ model. A and B: Representative images of the medial femoral-tibial joint of the knee joints from wildtype (WT) and Hybid ${ }^{-1-}$ mice at 2 weeks (A) and 4 weeks (B) after the surgery. Paraffin sections of the joints obtained from wild-type and $\mathrm{Hybid}^{-1-}$ mice were stained with hematoxylin and eosin (HE) and Safranin 0/Fast green for the analysis. C: Evaluation of the articular cartilage (Ca), synovium (Sy), and osteophyte (Os) of the knee joints. $O A$ changes in the knee joints, including articular cartilage, synovium, and bone, from wild-type and Hybid $^{-1-}$ mice were quantified in $\mathrm{HE}-$ and Safranin $0 /$ Fast green-stained sections, according to the histologic scoring system (Materials and Methods). Data are expressed as means \pm SD (C). $n=5$ each for WT and Hybid ${ }^{-1}$ mice (C). ${ }^{*} P<0.05$, $* * P<0.01$. Scale bars $=100 \mu \mathrm{m}(\mathbf{A}$ and $\mathbf{B})$. of both wild-type and $\mathrm{Hybid}^{-/-}$mice as compared with the sham-operated joint (Figure 2). The expression levels of inflammatory cytokines, including $I l 1 \beta, I l 6$, and Tnf, were higher by 15.5-fold, 9.2-fold, and 4.3-fold in wild-type mouse OA joint compared with wild-type mouse shamoperated joint, respectively (Figure 2). Although overexpression of these cytokines was observed in $\mathrm{Hybid}^{-/-}$ mouse OA joint, the levels of Ill $\beta$ and Tnf were significantly higher in the wild-type OA joint than in $\mathrm{Hybid}^{-/-}$ OA joint (Figure 2). Significantly increased expression of $T g f \beta$ in OA joint tissues from wild-type and $\mathrm{Hybid}^{-1-}$ mice compared with sham-operated joint tissues was also observed (Figure 2). More important, the $C d 31$ expression in Hybid $^{-1-}$ mouse OA joint was significantly lower than that in wild-type mouse OA joint (Figure 2), suggesting less vascularity in the $\mathrm{Hybid}^{-1-}$ mouse OA joint tissue compared with the wild-type OA joint tissue.

\section{Immunohistochemical Localization of Hybid in $\mathrm{OA}$ Joint Tissue}

Hybid was immunolocalized to cells in the synovium, chondrocytes in the articular cartilage, and osteophyte cells in OA knee joints of wild-type mice at 2 weeks after the 
surgery (Figure 3A). At 4 weeks after MCLT/M OA surgery, immunostained cells were observed in the synovium and osteophyte of wild-type mice, and chondrocytes in the articular cartilage were occasionally positive (Figure 3B). Only negligible background immunostaining was obtained with nonimmune $\operatorname{IgG}$ for the wild-type mouse joints at 2 and 4 weeks (Figure 3A) (data not shown for joint at 4 weeks). Similarly, there was only background immunostaining with anti-Hybid antibody or nonimmune $\operatorname{IgG}$ for $\mathrm{Hybid}^{-/-}$mouse joints at 2 weeks (Figure 3C) and at 4 weeks (Figure 3D) (data not shown for nonimmune $\mathrm{IgG}$ ).

\section{Decreased Blood Vessels at the Synovial and Periosteal Junction in Hybid $^{-/}$Mouse Knee Joints}

Histological vascularity at the synovial and periosteal junction of knee joint at 2 weeks after MCLT/M OA surgery appeared to be decreased in Hybid ${ }^{-1-}$ mice and the Cd31 expression level was significantly lower in joint tissue from
Hybid $^{-/-}$mice than in that from wild-type mice (Figure 2). The vascular density was determined by immunohistochemistry in paraffin sections of knee joint tissues using anti-CD31 antibody, which recognizes vascular endothelial cells. The number of blood vessels lined by CD31immunostained endothelial cells at the synovial and periosteal junction, around which osteophyte is formed, was significantly decreased in the Hybid ${ }^{-1-}$ mice compared with that in the wild-type mice (Figure 4).

\section{Accumulation of HMW-HA in Hybid ${ }^{-/-}$Mouse Knee Joints in MCLT/M OA Model}

Amounts and molecular weight distribution of HA in OA knee joint tissues were examined at 2 weeks after surgery. HA was extracted from the joint tissues of Hybid ${ }^{-1-}$ and wildtype mice, and then subjected to biochemical analyses. Amounts of HA were 23.9-fold higher in the samples from the Hybid $^{-/-}$mice $(2.39 \pm 0.78 \mu \mathrm{g} / \mathrm{mg})$ than in the wild-type
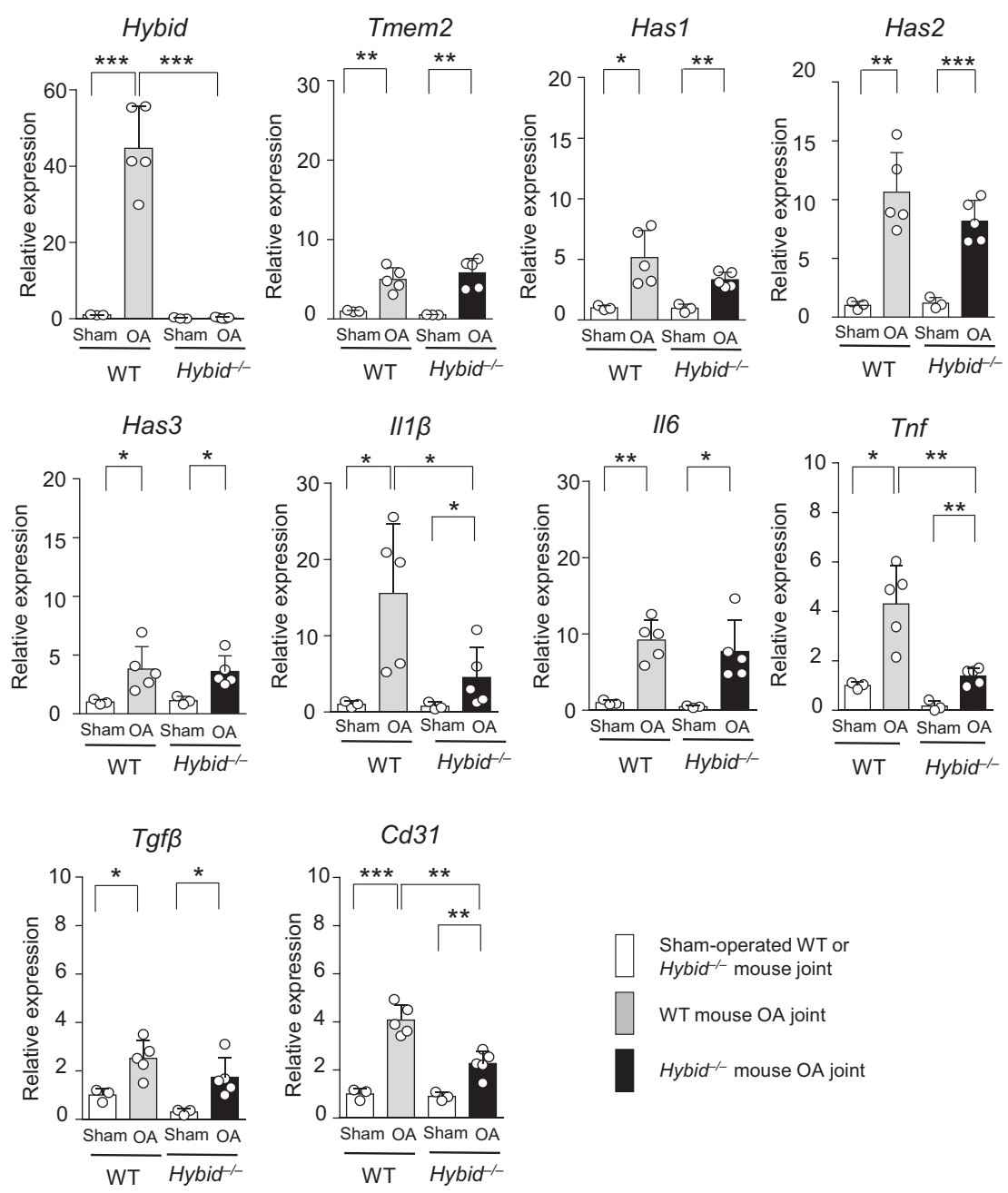

Figure 2 Expression of Hybid, Tmem2, Has1, Has2, Has3, Il1 $\beta$, Il6, Tnf, Tgf $\beta$, and Cd31 in knee joint tissues. Sham-operated and osteoarthritis (OA) joint tissues, including synovium, meniscus, and ligaments, were obtained from wild-type (WT) and $\mathrm{Hybid}^{-1-}$ mice at 2 weeks after the surgery; and mRNA was extracted. The relative expression of each target mRNA was determined by quantitative real-time $P C R$ by normalizing to the expression of Gapdh using $\Delta \Delta C_{T}$ method. The mean target gene/Gapdh ratio in wild-type mouse sham-operated joint is set at 1 . Data are expressed as means \pm SD. $n=3$ for sham-operated joint tissue; $n=5$ for $0 \mathrm{~A}$ joint tissue. ${ }^{*} P<0.05$, ${ }^{* *} P<0.01$, and ${ }^{* * *} P<0.001$. 
A

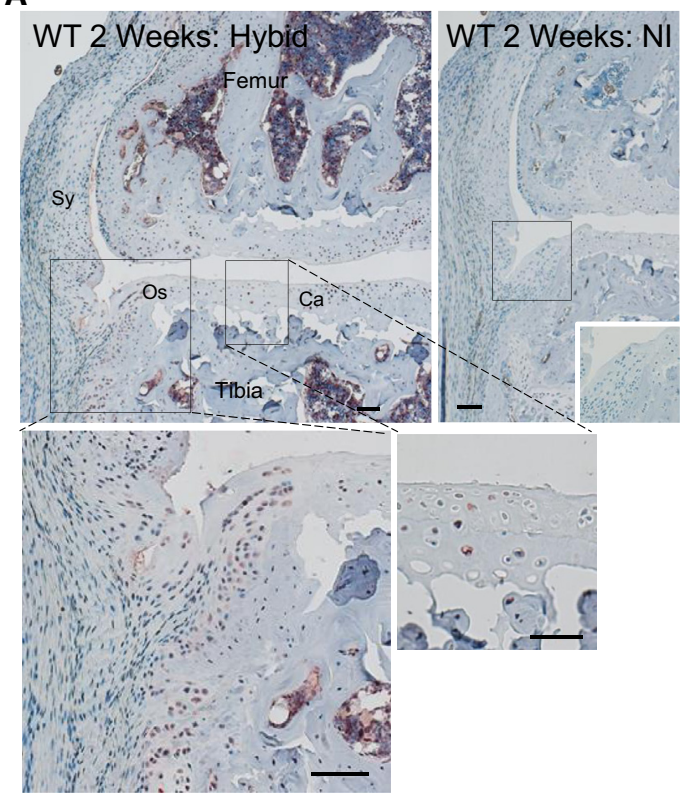

C

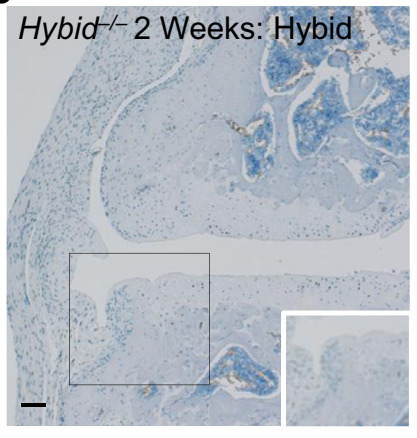

B

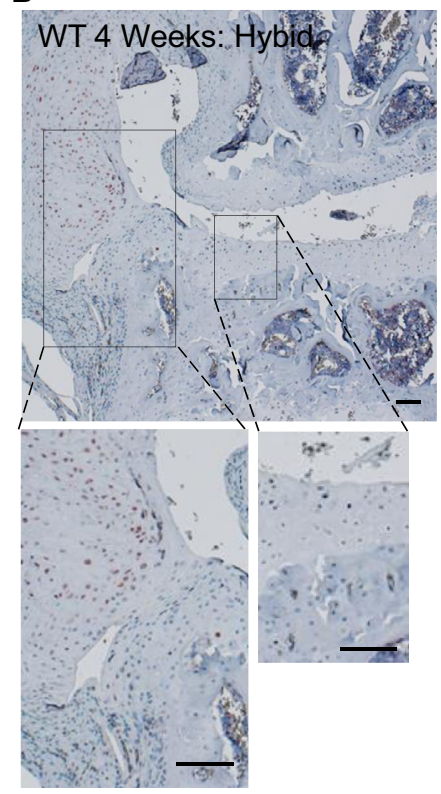

D

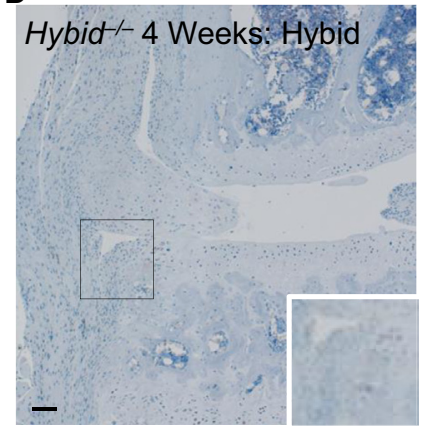

Figure 3 Immunolocalization of Hybid in knee joints of wild-type (WT) and $\mathrm{Hybid}^{-/-}$mice. Paraffin sections of knee joints obtained from WT and Hybid ${ }^{-} /-$mice at 2 weeks (A and $\mathbf{C}$ ) or 4 weeks (B and $\mathbf{D}$ ) after the surgery for medial collateral ligament transection with meniscus removal osteoarthritis model were immunostained with anti-Hybid antibody (Hybid) or nonimmune IgG (NI). Boxed areas are shown at higher magnification in the insets. Scale bars $=50 \mu \mathrm{m}(\mathbf{A}-\mathbf{D})$. Ca, cartilage; Os, osteophyte; Sy, synovium. mice $(0.10 \pm 0.003 \mu \mathrm{g} / \mathrm{mg})$ (Figure 5A). Size-exclusion chromatography using high-performance liquid chromatography and enzyme-linked immunosorbent assay for measurement of HA amounts showed a peak of HA molecular weight in the Hybid $^{-/}$joint samples at $>2500 \mathrm{kDa}$ (fraction 41 ), whereas it is approximately $1000 \mathrm{kDa}$ (fraction 47) in the wild-type samples (Figure $5 \mathrm{~B}$ ). The ratio of HA species $>1000 \mathrm{kDa}$ (fractions 33 to 47) to total HA (fractions 33 to 61) was significantly higher in the samples extracted from the Hybid $^{-/-}$joint tissue $(72.7 \% \pm 3.4 \%)$ compared with those from the wild-type samples $(54.0 \% \pm 4.7 \%)$ (Figure $5 \mathrm{C})$. The amounts and molecular weight distribution of HA in normal knee joint tissues from wild-type and $\mathrm{Hybid}^{-/}$mice were assessed at the age of 12 weeks. Both amounts and molecular weight distribution were similar in the wild-type and $\mathrm{Hybid}^{-/-}$mouse samples (Supplemental Figure S4). Interestingly, the HA amounts in $\mathrm{Hybid}^{-/-}$mice were higher in the OA tissue $(2.39 \pm 0.78 \mu \mathrm{g} / \mathrm{mg})$ than in the normal joint tissues $(0.78 \pm 0.08 \mu \mathrm{g} / \mathrm{mg})$, whereas the ratios of $\mathrm{HA}$ species at $>1000 \mathrm{kDa}$ in both $\mathrm{Hybid}^{-/}$and wild-type mice $(73.42 \% \pm 1.89 \%$ and $67.97 \% \pm 3.70 \%$, respectively) were similar to that in Hybid $^{-/-}$OA joint tissue $(72.7 \% \pm 3.4 \%)$.
Because Has1, Has2, and Has3 were up-regulated in OA joints of wild-type and $\mathrm{Hybid}^{-/-}$mice compared with shamoperated joints (Figure 2), these data suggest that the accumulation of HA with molecular weight $>1000 \mathrm{kDa}$ in $\mathrm{Hybid}^{-/-}$mouse OA joint is due to decreased depolymerization of overproduced HMW-HA mediated by the Has genes in the joint tissues.

\section{Inhibitory Effects of Intra-Articular HMW-HA Injections on OA Changes in Wild-Type Mouse Knee Joints in MCLT/M OA Model}

To test the effects of HMW-HA of $2700 \mathrm{kDa}$ on OA changes, HMW-HA was injected intra-articularly three times at 1, 2, and 3 weeks after surgery for MCLT/M OA model in wild-type mice, and histologically examined the joints at 1 week after the last intra-articular injection. Destruction of the articular cartilage and osteophyte formation appeared to be suppressed in the mouse group treated with HMW-HA compared with the control group, which received vesicle alone (Figure 6A). Scores of structure change and proteoglycan depletion of the articular 
A

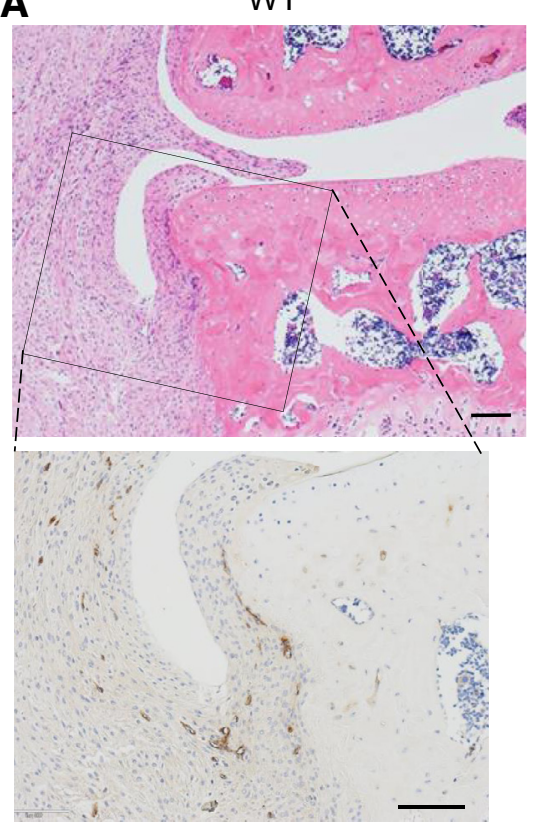

B
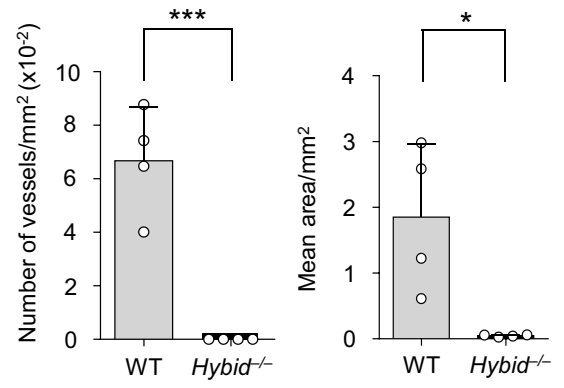

Figure 4 Decreased vascular density around osteophytes at the synovial and periosteal junction in the medial femoral-tibial joint of $\mathrm{Hybid}^{-/-}$ mice. A: Immunostaining of CD31 in the knee joints from wild-type (WT) and Hybid ${ }^{-1-}$ mice at 2 weeks after the surgery. The serial paraffin sections of the knee joints fixed using the AMeX method were stained with hematoxylin and eosin (HE) and immunostained with anti-CD31 antibody. Boxed regions in $\mathrm{HE}$-stained sections indicate the corresponding areas immunostained with antiCD31 antibody. Note the brown CD31immunostained blood vessels with lumen and CD31-immunostained structure. B: Quantification of number of CD31-positive vessels with lumen and mean area of CD31-positive structure at the synovial and periosteal junction, showing a marked decrease in vascular density in $\mathrm{Hybid}^{-/-}$ mice. Data are expressed as means \pm SD (B). $n=$ 4 (B). ${ }^{\star} P<0.05,{ }^{* * *} P<0.001$. Scale bars $=100$ $\mu \mathrm{m}(\mathbf{A})$. cartilage and those of osteophyte size and maturity were significantly decreased in the HMW-HA-treated group compared with the control group (Figure 6B). There were no changes in synovitis scores between the two groups (Figure 6B).

\section{Reduced Cartilage Destruction and 0steophyte Formation in $\mathrm{Hybid}^{-/-}$Mouse Knee Joints in DMM OA Model}

Because DMM OA model is considered to more closely resemble the slowly progressive human $\mathrm{OA},{ }^{6}$ the histologic changes of the knee joints were examined in the DMM OA model. Destruction of the articular cartilage and osteophyte formation of the tibia were observed in both $\mathrm{Hybid}^{-1-}$ and wild-type mice at 8 weeks after the surgery, and these changes progressed at 12 weeks (Figure 7A). When severity of the cartilage destruction and osteophyte formation was evaluated by scoring system, size and maturity of osteophyte were significantly decreased in $\mathrm{Hybid}^{-1-}$ mouse group than in wild-type mouse group (Figure 7A). The degree of structural change and proteoglycan depletion scores tended to be less severe in Hybid ${ }^{-1-}$ mouse group compared with wild-type mouse group, although not significantly different (Figure 7B). There were no changes in synovitis scores between the two groups (Figure 7B).

\section{Discussion}

This study demonstrates, to the best of our knowledge, for the first time, that destruction of articular cartilage in mechanical stress-induced OA models is inhibited in Hybid $^{-/-}$mice compared with that in wild-type mice. Yamamoto et $\mathrm{al}^{16}$ reported that Tmem 2 acts as an essential cell-surface hyaluronidase in developing mice, showing the ubiquitous expression in adult mouse tissues, including the articular cartilage and synovium. In the present study, although Hybid was remarkably up-regulated in the wildtype mouse OA joint tissue, the Tmem 2 expression levels were almost similar in the wild-type and $\mathrm{Hybid}^{-1-}$ OA joints. Previous studies have provided the data that HYBID, but not TMEM2, overproduced by chondrocytes and 

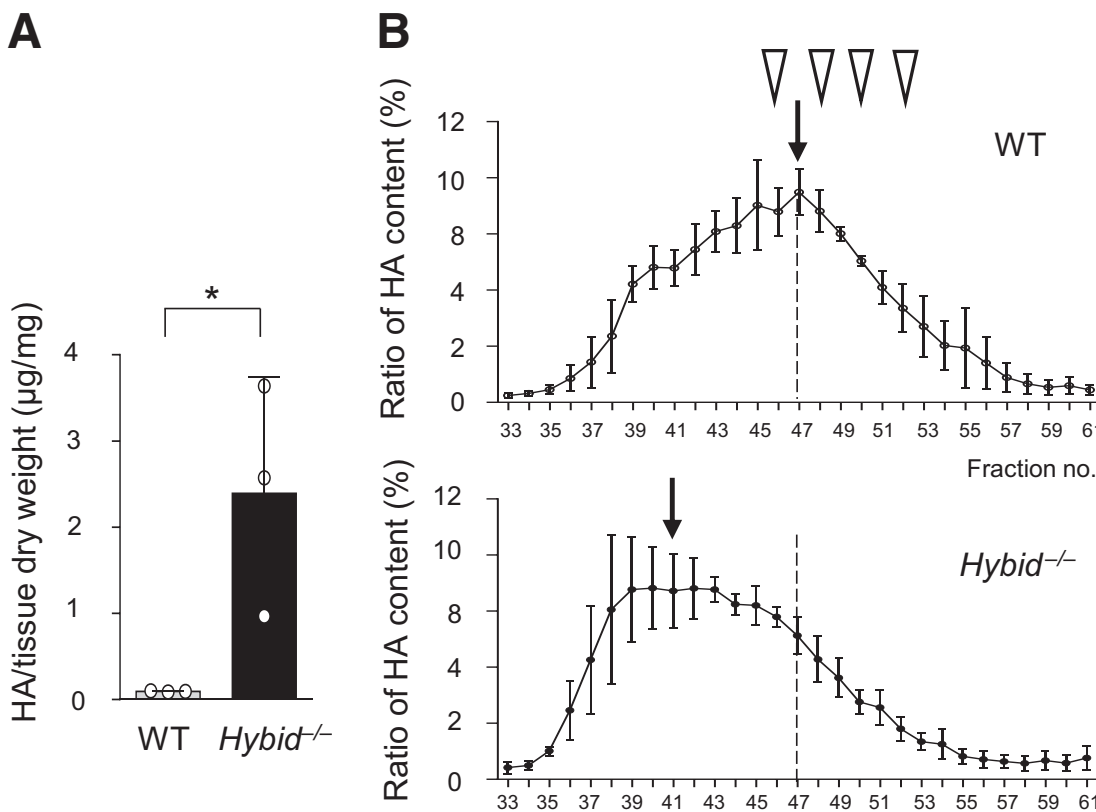

C
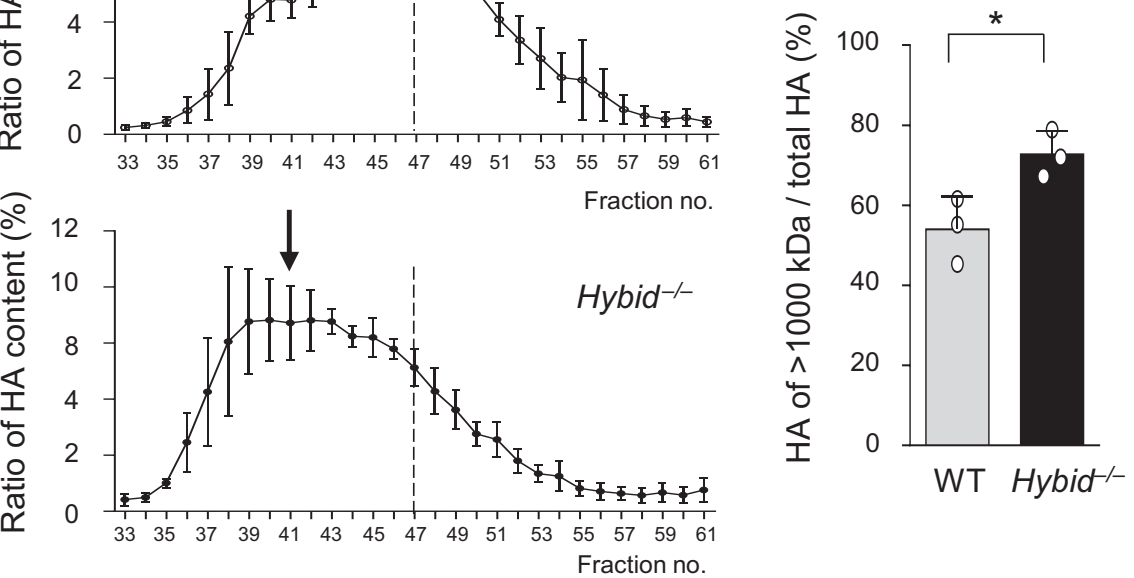

Figure 5 Amounts and size distribution of hyaluronan (HA) in the knee joint tissues of wild-type (WT) and Hybid ${ }^{-1-}$ mice at 2 weeks after surgery for medial collateral ligament transection with meniscus removal osteoarthritis model. A: Total amount of HA per joint tissue. Knee joint tissues were obtained from WT and Hybid ${ }^{-1-}$ mice at 2 weeks after the surgery, and HA was extracted from the tissues. Total amount of HA per dry tissue weight was calculated. B: Determination of HA size distribution in the wild-type or Hybid ${ }^{-1}$ mouse joint tissues obtained after the surgery. HA extracted from the joint tissues of wild-type and $\mathrm{Hybid}^{-1-}$ mice was subjected to size-exclusion chromatography, and HA concentrations in each fraction were determined by enzyme-linked immunosorbent assay. Arrows indicate the peak fraction of the wild-type and Hybid ${ }^{-1}$ mouse joint tissues; dashed line, fraction containing HA of approximately $1000 \mathrm{kDa}$; arrowheads, elution peaks of NaHA with 2012, 887, 222, and $55 \mathrm{kDa}$ from left to right. C: Ratios of HA species of molecular weight $>1000 \mathrm{kDa} /$ total HA in the wild-type and Hybid ${ }^{-1-}$ mouse joint tissues. Data are expressed as means \pm SD (A-C). $n=3$ each for WT and $H_{y b i d^{-1-}}$ mice $(\mathbf{A}-\mathbf{C}) .{ }^{*} P<0.05$

synovial fibroblasts in human OA joint is implicated for HA degradation of the $\mathrm{OA}$ articular cartilage and depolymerization of HMW-HA into lower-molecular-weight HA in OA synovial fluid, respectively. ${ }^{17,18}$ Considering the findings of these studies together, like human OA, Hybid seems to play a key role in the progressive cartilage destruction by acting as hyaluronidase in mouse OA joint. This study, however, does not completely exclude the possibility that mildly up-regulated Tmem 2 in joint tissues is also involved in the OA changes of both wild-type and $\mathrm{Hybid}^{-1-}$ mice.

Histologic studies on human OA cartilage have shown that destruction of the articular cartilage is initiated by degradation of the HA-aggrecan network before digestion of collagen fibrils. ${ }^{3,7}$ Collagenolytic MMPs, such as MMP-1, MMP-13, and MMP-14, play a major role in cleavage of cartilage major collagen (ie, type II collagen), and gelatinolytic activities of MMPs, including MMP-2, MMP-3, MMP-7, and MMP-9, and other proteinases are responsible for further degradation of gelatins generated by denaturation of collagens cleaved by the collagenolytic MMPs at body temperature. ${ }^{7}$ Previous studies have demonstrated that ADAMTS5, but not ADAMTS4, is a key enzyme for aggrecan degradation in mouse arthritis on the basis of the data that OA changes were inhibited in only ADAMTS5deficient mice. ${ }^{26,27}$ In mechanical stress-induced OA mouse models, ADAMTS5 and MMP-13 expressed in the articular cartilage are considered to play a central role in degradation of aggrecan and collagens, leading to cartilage destruction. ${ }^{5,26-28}$ Concerning HA degradation, however, only limited information is available. Although Deroyer et $\mathrm{al}^{29}$ recently reported immunohistochemical expression of Hybid by articular chondrocytes in murine OA model and proposed a function of this molecule as profibrotic mediator, the authors did not study the action of Hybid on HA degradation. The present study demonstrates that in MCLT/ M OA model, destruction of the articular cartilage in $\mathrm{Hybid}^{-/-}$mice is inhibited and this inhibition is associated with accumulation of HMW-HA in the joint tissues. In addition, intra-articular injections of HMW-HA to wild-type mouse joint in the early stage of MCLT/M OA attenuated the cartilage destruction. Altogether, these data indicate the possibility that Hybid plays a key role in the initial step of the cartilage destruction through HA depolymerization of the HA-aggrecan network, and suggest that HMW-HA may contribute to protection of articular cartilage from destruction in the early stage of OA.

Human HYBID was originally identified by microarray analysis as a molecule capable of binding to and degrading $\mathrm{HA}$ from the candidate genes, which were up-regulated and down-regulated by treatment with histamine and TGF- $\beta$, respectively. ${ }^{10}$ In addition, TNF- $\alpha$ and IL- 6 promote the HYBID expression in human OA chondrocytes ${ }^{17}$ and 
A
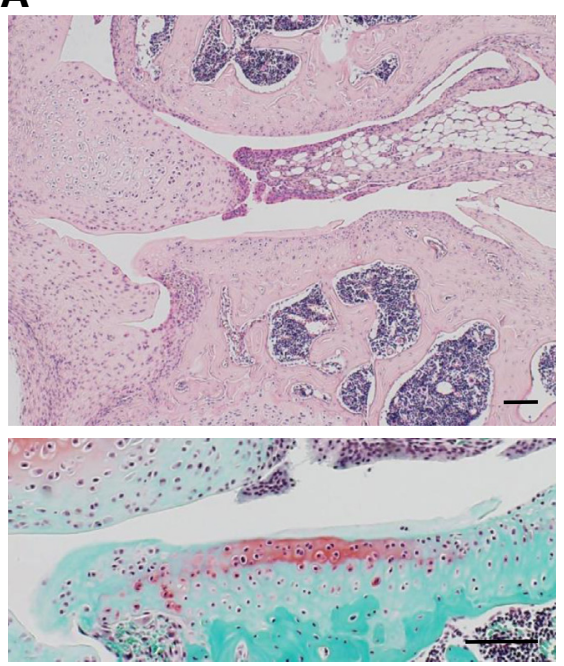
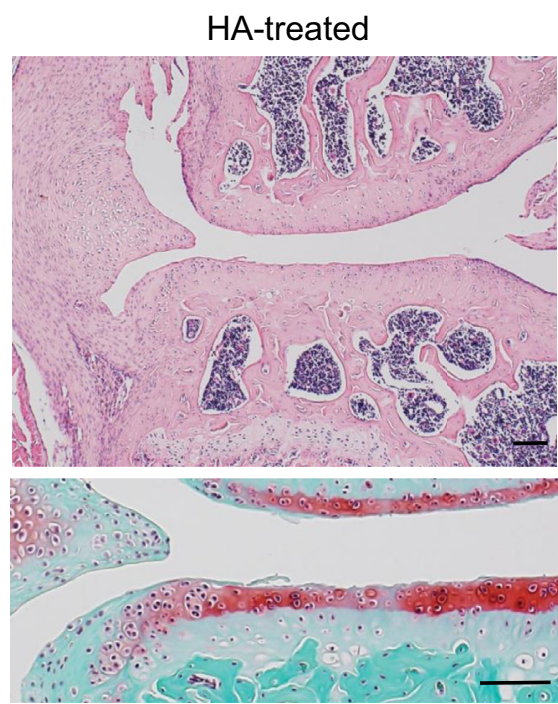

B
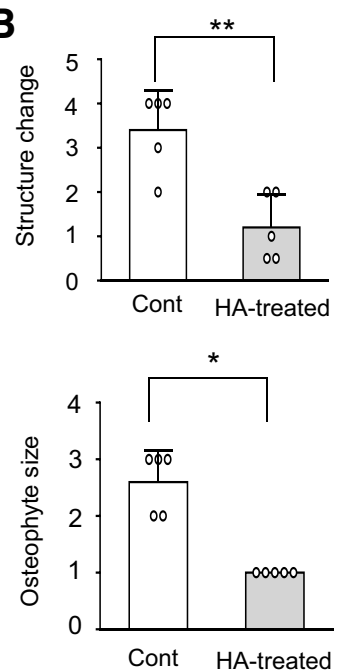
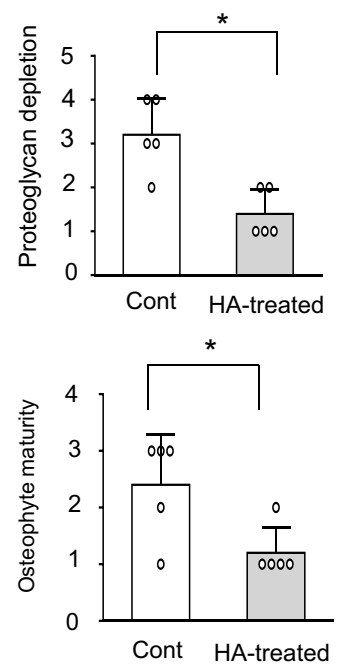

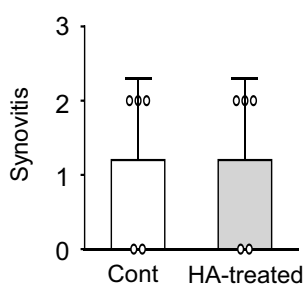

Figure 6 Intra-articular injection of highmolecular-weight hyaluronan (HMW-HA) reverses osteoarthritis $(O A)$ changes in medial collateral ligament transection with meniscus removal 0 A model. A: Representative histologic images of the knee joints from wild-type mice treated with vehicle (Control) or HMW-HA (HAtreated). At 1 week after the surgery of both knee joints, the left and right knee joints received injections of HMW-HA and saline (10 $\mu \mathrm{L}$ each), respectively, three times every week. At 1 week after the last injection, the joints were removed and subjected to histologic examination with hematoxylin and eosin and Safranin 0/Fast green staining. B: Evaluation of the articular cartilage, synovium, and osteophyte of the knee joints. OA changes in the knee joints, including articular cartilage, synovium, and bone, from control (Cont) and HMW-HA injected (HA-treated) mice were quantified by histologic scoring system (Materials and Methods). Data are expressed as means $\pm \mathrm{SD}$ (B). $n=5$ (A and $\mathbf{B}) .{ }^{*} P<0.05$, ${ }^{* * P}<0.01$. Scale bars $=100 \mu \mathrm{m}(\mathbf{A})$. synovial fibroblasts, ${ }^{18}$ respectively, and IL-6 stimulates the expression in OA chondrocytes (Jun Shiozawa et al, unpublished data). The present study showed that the mRNA expression of both Tnf and Il6 was enhanced in OA joint tissue. The expression of Tnf and Il6 is known to be induced by increased mechanical stress (ie, hydrostatic pressure) to cultured chondrocytes. ${ }^{30}$ In addition, IL-1 $\beta$, which stimulates Il6 expression in chondrocytes, ${ }^{31}$ was also highly expressed in our OA model. Thus, these factors induced by increased mechanical stress in the articular cartilage may be responsible for the up-regulation of Hybid in this model. On the other hand, previous study on human colon carcinoma cells pointed out that $H Y B I D$ is up-regulated by hypoxia-inducible factor $2 \alpha$ (HIF-2 $\alpha$ ) through its direct binding to the hypoxia responsible element of the promoter, ${ }^{13}$ and the Hif $2 \alpha$ expression is increased by the articular cartilage in mouse mechanical stress-induced OA models. ${ }^{32,33}$ Therefore, it seems likely that Hif- $2 \alpha$ may be also involved in upregulation of Hybid in this model. However, because no information is available for the hypoxia responsible element in the promoter of the mouse Hybid gene, further studies are necessary to support this hypothesis.

One of the important messages in the present study is that size and maturity of osteophytes in $\mathrm{Hybid}^{-/}$mice were significantly reduced in both MCLT/M and DMM OA models. In the MCLT/M OA model, these findings were associated with accumulation of HMW-HA in the joint tissue of $\mathrm{Hybid}^{-/-}$mice, and osteophyte formation was decreased by treatment with HMW-HA in wild-type OA mice. These data strongly suggest that the inhibitory effect on osteophyte formation is ascribed to HMW-HA maintained in the knee joints. Fully developed osteophyte is composed of cartilage and bone parts, and the cartilage part is formed mainly by proliferation of mesenchymal stem cells stimulated with TGF- $\beta$, whereas bone morphogenic protein 2 may be important for initiation of the bone formation via endochondral ossification. ${ }^{34}$ Roelofs et $\mathrm{al}^{35}$ have recently reported that $P d g f r \alpha+G d f 5$-lineage progenitors, containing subsets of Prg4-expressing progenitors and Sox9-expressing progenitors, both of which are resident 


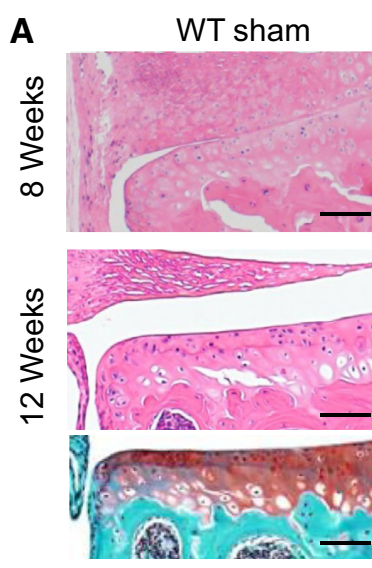

B

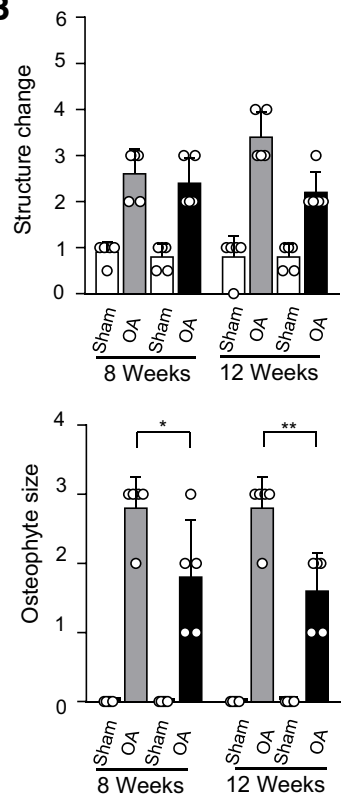

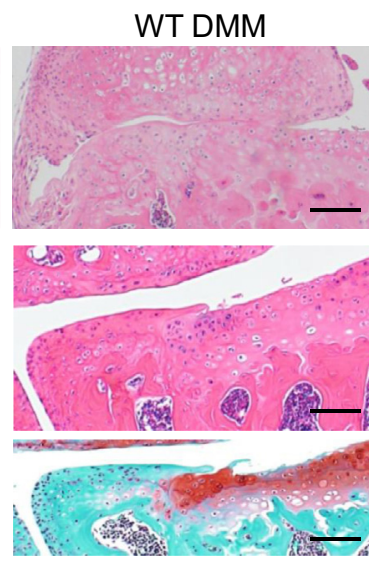
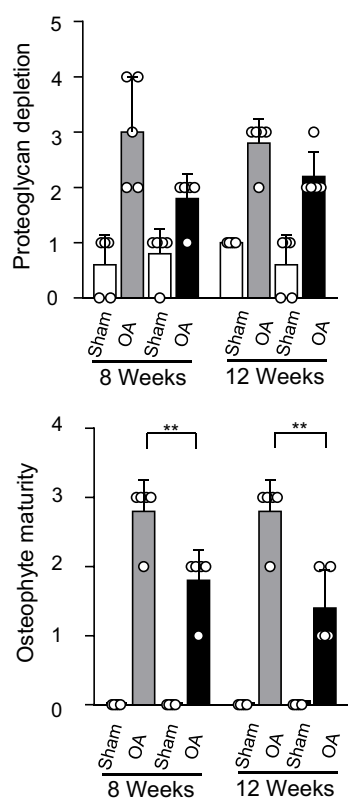
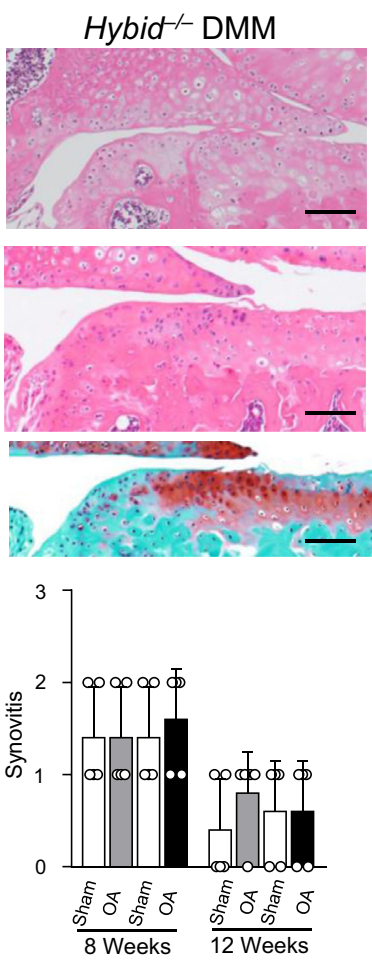

Sham-operated WT or Hybid $^{--}$mouse joint

WT mouse OA joint
Figure 7 Histologic evaluation of the knee joints in destabilization of the medial meniscus (DMM) osteoarthritis (OA) model. A: Representative images of the medial femoral-tibial joint from wild-type (WT) and $\mathrm{Hybid}^{-/-}$mice at 8 and 12 weeks after the sham operation or the surgery for DMM. Paraffin sections of the joints obtained from wild-type and $\mathrm{Hybid}^{-1}$ mice were stained with hematoxylin and eosin and Safranin 0/Fast green for the analysis. B: Evaluation of the articular cartilage, synovium, and osteophyte of the knee joints. OA changes in the knee joints, including articular cartilage, synovium, and bone, from wildtype and $\mathrm{Hybid}^{-/-}$mice were quantified by histologic scoring system (Materials and Methods). Data are expressed as means \pm SD (B). $n=5$ each for WT and Hybid ${ }^{-1-}$ mice (A and $\left.\mathbf{B}\right)$. ${ }^{*} P<0.05$, ${ }^{* *} P<0.01$. Scale bars $=200 \mu \mathrm{m}(\mathbf{A})$. at the junction of periosteum and synovium near the articular cartilage, are activated in $\mathrm{OA}$ to form osteophyte cartilage and bone parts, respectively. The molecular mechanisms of reduced osteophyte formation in OA models of $\mathrm{Hybid}^{-/-}$mice are not clear at present. However, the current study has provided the data that vascular density in the tissue at the synovial and periosteal junction, which is adjacent to osteophyte, was significantly decreased in $\mathrm{Hybid}^{-/-}$mice compared with wild-type mice. Lowermolecular-weight HA, especially HA oligosaccharides, is well known to exhibit proangiogenic capability, whereas HMW-HA functions as anti-angiogenic agent. ${ }^{36,37}$ Previous study on the developing $\mathrm{Hybid}^{-/-}$mice has demonstrated that vascular endothelial growth factor-mediated angiogenesis in the growth plate of long bones is suppressed by the action of HMW-HA accumulated in the growth plate. ${ }^{15}$ In addition, HMW-HA is reported to have an effect on maintaining stemness of cancer stem cells and osteoprogenitor cells. ${ }^{38,39}$ Therefore, Hybid-mediated depolymerization of HMW-HA into lower-molecular-weight $\mathrm{HA}$ in wild-type $\mathrm{OA}$ mice may facilitate to provide nutrient and oxygen required for the $G d f 5$-positive mesenchymal stem cells to proliferate and differentiate to chondrocytes and osteoblasts at the synovial and periosteal junction, although further studies are necessary to support this hypothesis.

In summary, this study demonstrates, for the first time, that destruction of the articular cartilage and osteophyte formation in the mechanical stress-induced OA models are inhibited in $\mathrm{Hybid}^{-/-}$mice. Previous studies have shown that HYBID is highly produced by chondrocytes located in the HA-depleted zone of the articular cartilage and synovial fibroblasts in human OA knee joints. ${ }^{17,18}$ These data suggested that HYBID plays a dual role in destruction of the articular cartilage through the two different pathways: the direct cartilage destruction due to HA depolymerization of the HA-aggrecan framework in the cartilage ECM and the indirect destruction secondary to enhanced mechanical stress to the articular cartilage caused by decreased shockabsorber function of HMW-HA in synovial fluid. ${ }^{17,18}$ However, these are correlation studies using the tissues and synovial fluid samples derived from patients with advanced knee OA, and thus effect of HYBID-mediated HA 
depolymerization on OA progression could not be clarified. The present study provides the data that HMW-HA accumulated in the OA joint tissues of Hybid ${ }^{-1}$ mice or supplemented by injections into OA knee joints of wild-type mice inhibits cartilage destruction and osteophyte formation. HMW-HA is known to be anti-inflammatory and anti-angiogenic, and act as a scavenger for reactive oxygen species. ${ }^{40,41}$ Thus, the data in the present study, together with previously reported functions of HMW-HA, suggest that Hybid-mediated HA depolymerization is implicated for the progression of mechanical stress-induced knee OA. Although the present study showed that repeated intraarticular injections of HMW-HA started at 1 week after the operation are effective to attenuate OA changes and osteophyte formation, this condition of the injection may not be clinically applicable because a limited number of early-stage knee OA patients consult clinicians. Because previous and preliminary studies showed that IL-6 stimulates the HYBID expression in OA synovium ${ }^{18}$ and chondrocytes (Jun Shiozawa et al, unpublished data), therapeutic strategies using IL-6 receptor blockade, such as tocilizumab or inhibitors to HYBID activity, may be useful for suppression of $\mathrm{OA}$ progression, although further analyses on the characteristics and role of HYBID in pathophysiological conditions are necessary.

\section{Author Contributions}

M.M. acquired, analyzed, and interpreted the data, and drafted the article; S.d.V., H.K., and C.Y. acquired, analyzed, and interpreted the data; M.S., T.N., Y.E., and H.Y. assisted in the experiments and analyzed and interpretated the data; K.K. performed conception and design; M.I. performed conception, design, and drafting the article; Y.O. performed conception, design, and drafting the article, had a full access to all the data in the study, and takes responsibility for the integrity of the data and the accuracy of the data analysis; all authors read and approved the final manuscript.

\section{Supplemental Data}

Supplemental material for this article can be found at http://doi.org/10.1016/j.ajpath.2021.07.008.

\section{References}

1. Hunter DJ, Bierma-Zeinstra S: Osteoarthritis. Lancet 2019, 393: 1745-1759

2. Felson DT: Osteoarthritis as a disease of mechanics. Osteoarthritis Cartilage 2013, 21:10-15

3. Primorac D, Molnar V, Rod E, Jelec Z, Cukelj F, Matisic V, Vrdoljak T, Hudetz D, Hajsok H, Boric I: Knee osteoarthritis: a review of pathogenesis and state-of-the-art non-operative therapeutic considerations. Genes (Basel) 2020, 11:854

4. Kuyinu EL, Narayanan G, Nair LS, Laurencin CT: Animal models of osteoarthritis: classification, update, and measurement of outcomes. J Orthop Surg Res 2016, 11:19
5. Kamekura S, Hoshi K, Shimoaka T, Chung U, Chikuda H, Yamada T, Uchida M, Ogata N, Seichi A, Nakamura K, Kawaguchi H: Osteoarthritis development in novel experimental mouse models induced by knee joint instability. Osteoarthritis Cartilage 2005, 13:632-641

6. Glasson SS, Blanchet TJ, Morris EA: The surgical destabilization of the medial meniscus (DMM) model of osteoarthritis in the 129/SvEv mouse. Osteoarthritis Cartilage 2007, 15:1061-1069

7. Okada Y: Proteinases and matrix degradation. Edited by Firestein GS, Budd RC, Gabriel SE, McInnes IB, R O'Dell J. In Kelley and Firestein's Textbook of Rheumatology. ed 10. Philadelphia, PA: Elsevier Inc., 2017. pp. 106-125

8. Csoka AB, Frost GI, Stern R: The six hyaluronidase-like genes in the human and mouse genomes. Matrix Biol 2001, 20:499-508

9. Harada H, Takahashi M: CD44-dependent intracellular and extracellular catabolism of hyaluronic acid by hyaluronidase-1 and -2 . J Biol Chem 2007, 282:5597-5607

10. Yoshida H, Nagaoka A, Kusaka-Kikushima A, Tobiishi M, Kawabata K, Sayo T, Sakai S, Sugiyama Y, Enomoto H, Okada Y, Inoue S: KIAA1199, a deafness gene of unknown function, is a new hyaluronan binding protein involved in hyaluronan depolymerization. Proc Natl Acad Sci USA 2013, 110:5612-5617

11. Nagaoka A, Yoshida H, Nakamura S, Morikawa T, Kawabata K Kobayashi M, Sakai S, Takahashi Y, Okada Y, Inoue S: Regulation of hyaluronan (HA) metabolism mediated by HYBID (hyaluronanbinding protein involved in HA depolymerization, KIAA1199) and HA synthases in growth factor-stimulated fibroblasts. J Biol Chem 2015, 290:30910-30923

12. Abe S, Usami S, Nakamura Y: Mutations in the gene encoding KIAA1199 protein, an inner-ear protein expressed in Deiters' cells and the fibrocytes, as the cause of nonsyndromic hearing loss. J Hum Genet 2003, 48:564-570

13. Evensen NA, Li Y, Kuscu C, Liu J, Cathcart J, Banach A, Zhang Q, Li E, Joshi S, Yang J, Denoya PI, Pastorekova S, Zucker S, Shroyer KR, Cao J: Hypoxia promotes colon cancer dissemination through up-regulation of cell migration-inducing protein (CEMIP). Oncotarget 2015, 6:20723-20739

14. Yoshida H, Nagaoka A, Nakamura S, Sugiyama Y, Okada Y, Inoue S Murine homologue of the human KIAA1199 is implicated in hyaluronan binding and depolymerization. FEBS Open Bio 2013, 3:352-356

15. Shimoda M, Yoshida H, Mizuno S, Hirozane T, Horiuchi K, Yoshino Y, Hara H, Kanai Y, Inoue S, Ishijima M, Okada Y: Hyaluronan-binding protein involved in hyaluronan depolymerization controls endochondral ossification through hyaluronan metabolism. Am J Pathol 2017, 187:1162-1176

16. Yamamoto $H$, Tobisawa $\mathrm{Y}$, Inubushi $\mathrm{T}$, Irie $\mathrm{F}$, Ohyama $\mathrm{C}$, Yamaguchi Y: A mammalian homolog of the zebrafish transmembrane protein 2 (TMEM2) is the long-sought-after cell-surface hyaluronidase. J Biol Chem 2017, 292:7304-7313

17. Shimizu H, Shimoda M, Mochizuki S, Miyamae Y, Abe H, Chijiiwa M, Yoshida H, Shiozawa J, Ishijima M, Kaneko K, Kanaji A, Nakamura M, Toyama Y, Okada Y: Hyaluronan-binding protein involved in hyaluronan depolymerization is up-regulated and involved in hyaluronan degradation in human osteoarthritic cartilage. Am J Pathol 2018, 188:2109-2119

18. Shiozawa J, de Vega S, Cilek MZ, Yoshinaga C, Nakamura T, Kasamatsu S, Yoshida H, Kaneko H, Ishijima M, Kaneko K, Okada Y: Implication of HYBID (hyaluronan-binding protein involved in hyaluronan depolymerization) in hyaluronan degradation by synovial fibroblasts in patients with knee osteoarthritis. Am J Pathol 2020, 190:1046-1058

19. Glasson SS, Chambers MG, Van Den Berg WB, Little CB: The OARSI histopathology initiative - recommendations for histological assessments of osteoarthritis in the mouse. Osteoarthritis Cartilage 2010, 18(Suppl):S17-S23

20. Lewis JS, Hembree WC, Furman BD, Tippets L, Cattel D, Huebner JL, Little D, DeFrate LE, Kraus VB, Guilak F, Olson SA: 
Acute joint pathology and synovial inflammation is associated with increased intra-articular fracture severity in the mouse knee. Osteoarthritis Cartilage 2011, 19:864-873

21. Kaneko H, Ishijima M, Futami I, Tomikawa-Ichikawa N, Kosaki K, Sadatsuki R, Yamada Y, Kurosawa H, Kaneko K, ArikawaHirasawa E: Synovial perlecan is required for osteophyte formation in knee osteoarthritis. Matrix Biol 2013, 32:178-187

22. Guide for the Care and Use of Laboratory Animals: Eighth Edition. Washington, DC, National Academies Press. Committee for the Update of the Guide for the Care and Use of Laboratory Animals; National Research Council, 2011

23. Sato Y, Mukai K, Watanabe S, Goto M, Shimosato Y: The AMeX method: a simplified technique of tissue processing and paraffin embedding with improved preservation of antigens for immunostaining. Am J Pathol 1986, 125:431-435

24. Chijiiwa M, Mochizuki S, Kimura T, Abe H, Tanaka Y, Fujii Y, Shimizu H, Enomoto H, Toyama Y, Okada Y: CCN1 (Cyr61) is overexpressed in human osteoarthritic cartilage and inhibits ADAMTS-4 (aggrecanase 1) activity. Arthritis Rheum 2015, 67: $1557-1567$

25. Tobiishi M, Sayo T, Yoshida H, Kusaka A, Kawabata K, Sugiyama Y, Ishikawa $\mathrm{O}$, Inoue $\mathrm{S}$ : Changes in epidermal hyaluronan metabolism following UVB irradiation. J Dermatol Sci 2011, 64:31-38

26. Glasson SS, Askew R, Sheppard B, Carito B, Blanchet T, Ma HL, Flannery CR, Peluso D, Kanki K, Yang Z, Majumdar MK, Morris EA: Deletion of active ADAMTS5 prevents cartilage degradation in a murine model of osteoarthritis. Nature 2005, 434: 644-648

27. Stanton H, Rogerson FM, East CJ, Golub SB, Lawlor KE, Meeker CT, Little CB, Last K, Farmer PJ, Campbell IK, Fourie AM, Fosang AJ: ADAMTS5 is the major aggrecanase in mouse cartilage in vivo and in vitro. Nature 2005, 434:648-652

28. Little CB, Barai A, Burkhardt D, Smith SM, Fosang AJ, Werb Z, Shah M, Thompson EW: Matrix metalloproteinase 13-deficient mice are resistant to osteoarthritic cartilage erosion but not chondrocyte hypertrophy or osteophyte development. Arthritis Rheum 2009, 60: $3723-3733$

29. Deroyer C, Charlier E, Neuville S, Malaise O, Gillet P, Kurth W, Chariot A, Malaise M, de Seny D: CEMIP (KIAA1199) induces a fibrosis-like process in osteoarthritic chondrocytes. Cell Death Dis 2019, 10:103

30. Takahashi K, Kubo T, Arai Y, Kitajima I, Takigawa M, Imanishi J, Hirasawa Y: Hydrostatic pressure induces expression of interleukin 6 and tumour necrosis factor alpha mRNAs in a chondrocyte-like cell line. Ann Rheum Dis 1998, 57:231-236

31. Henrotin YE, De Groote DD, Labasse AH, Gaspar SE, Zheng SX, Geenen VG, Reginster JY: Effects of exogenous IL-1 beta, TNF alpha, IL-6, IL-8 and LIF on cytokine production by human articular chondrocytes. Osteoarthritis Cartilage 1996, 4:163-173

32. Saito T, Fukai A, Mabuchi A, Ikeda T, Yano F, Ohba S, Nishida N, Akune T, Yoshimura N, Nakagawa T, Nakamura K, Tokunaga K, Chung UI, Kawaguchi H: Transcriptional regulation of endochondral ossification by HIF-2alpha during skeletal growth and osteoarthritis development. Nat Med 2010, 16:678-686

33. Yang S, Kim J, Ryu JH, Oh H, Chun CH, Kim BJ, Min BH, Chun JS: Hypoxia-inducible factor-2alpha is a catabolic regulator of osteoarthritic cartilage destruction. Nat Med 2010, 16:687-693

34. van der Kraan PM, van den Berg WB: Osteophytes: relevance and biology. Osteoarthritis Cartilage 2007, 15:237-244

35. Roelofs AJ, Kania K, Rafipay AJ, Sambale M, Kuwahara ST, Collins FL, Smeeton J, Serowoky MA, Rowley L, Wang H, Gronewold R, Kapeni C, Mendez-Ferrer S, Little CB, Bateman JF, Pap T, Mariani FV, Sherwood J, Crump JG, De Bari C: Identification of the skeletal progenitor cells forming osteophytes in osteoarthritis. Ann Rheum Dis 2020, 79:1625-1634

36. West DC, Hampson IN, Arnold F, Kumar S: Angiogenesis induced by degradation products of hyaluronic acid. Science 1985, 228: $1324-1326$

37. Takahashi Y, Li L, Kamiryo M, Asteriou T, Moustakas A, Yamashita H, Heldin P: Hyaluronan fragments induce endothelial cell differentiation in a CD44- and CXCL1/GRO1-dependent manner. J Biol Chem 2005, 280:24195-24204

38. Chanmee T, Ontong P, Kimata K, Itano N: Key roles of hyaluronan and its CD44 receptor in the stemness and survival of cancer stem cells. Front Oncol 2015, 5:180

39. Asparuhova MB, Chappuis V, Stahli A, Buser D, Sculean A: Role of hyaluronan in regulating self-renewal and osteogenic differentiation of mesenchymal stromal cells and pre-osteoblasts. Clin Oral Investig 2020, 24:3923-3937

40. Cyphert JM, Trempus CS, Garantziotis S: Size matters: molecular weight specificity of hyaluronan effects in cell biology. Int J Cell Biol 2015, 2015:563818

41. Tavianatou AG, Caon I, Franchi M, Piperigkou Z, Galesso D, Karamanos NK: Hyaluronan: molecular size-dependent signaling and biological functions in inflammation and cancer. FEBS J 2019, 286: 2883-2908 